Mots. Les langages du politique

$111 \mid 2016$

Normes et usages de la langue en politique

\title{
Les anglicismes dans les discours politiques au Québec et au Canada
}

Anglicisms in political discourse in Quebec and Canada

Los anglicismos en los discursos políticos en Quebec y en Canadá

Chantal Gagnon et Louise Brunette

\section{OpenEdition}

Journals

Édition électronique

URL : https://journals.openedition.org/mots/22316

DOI : $10.4000 /$ mots. 22316

ISBN : 978-2-84788-836-2

ISSN : 1960-6001

Éditeur

ENS Éditions

\section{Édition imprimée}

Date de publication : 10 septembre 2016

Pagination : 15-32

ISBN : 978-2-84788-835-5

ISSN : 0243-6450

Référence électronique

Chantal Gagnon et Louise Brunette, "Les anglicismes dans les discours politiques au Québec et au Canada », Mots. Les langages du politique [En ligne], 111 | 2016, mis en ligne le 10 septembre 2018, consulté le 23 avril 2022. URL : http://journals.openedition.org/mots/22316 ; DOI : https://doi.org/ $10.4000 /$ mots.22316

\section{(C) ENS Éditions}




\section{Les anglicismes dans les discours politiques au Québec et au Canada}

Au Québec, territoire dont la langue officielle est le français, la qualité de la langue constitue un perpétuel sujet d'actualité dans les médias et dans les instances. On s'interroge, par exemple, sur la qualité du français chez les jeunes, chez les enseignants et même chez les politiques. Pour les francophones actifs en politique, la question de la langue française n'est jamais simple, puisqu'il leur faut notamment trouver un juste équilibre entre la langue normalisée et la langue de la population. En d'autres mots, il s'agit pour eux de répondre aux normes de leur communauté linguistique. Comme l'explique Christian Le Bart (1998) :

Ainsi la communauté politique s'apparente-t-elle à une communauté linguistique au sens de Labov : non que tous parlent la même langue, mais parce que tous partagent «les mêmes normes quant à la langue». Les politiques ne sont pas contraints à parler comme tout le monde; ils sont contraints à parler comme tout le monde s'attend à les entendre parler, ce qui, pour la majorité d'entre eux (dominants culturels), ne signifie rien d'autre que l'obligation d'être soi-même.

Au Québec, le phénomène de contact des langues a des répercussions sur la norme. En effet, encerclés par les communautés d'expression anglaise de toute l'Amérique du Nord, les Québécois francophones sont en situation minoritaire sur leur continent (où ils comptent pour $2 \%$ à peine) et dans leur pays. Au quotidien, le contact des langues produit des effets qui donnent lieu à des opinions partagées, comme en font foi les commentaires politiques cidessous (notre mise en relief, ci-dessous et dans tous les autres exemples) :

[L]a vie de Brian Topp ressemble à un long déménagement qui se poursuivra, si son souhait se réalise, par une vie nouvelle à Ottawa, dans la résidence officielle du chef de l'opposition, après le 24 mars. Né à Longueuil d'un père anglophone originaire de l'Estrie et d'une mère francophone [...], M. Topp s'exprime parfaitement dans les deux langues, même s'il assortit son français de quelques anglicismes au passage. (Giroux, 2011, p.12) 
En soirée, avec des militants de Québec, [Philippe Couillard] a prononcé son discours sans télésouffleur, à l'aide de notes inscrites sur [des] feuilles qu'il a à peine consultées. Son niveau de langage était nettement plus familier qu'à l'habitude. Des anglicismes, qu'il ne faisait jamais entendre, se sont glissés ici et là. Le député d'Outremont a beaucoup parlé de hockey, il a rappelé son expérience de défenseur et il a même évoqué d'anciennes blessures. Si bien que le fil blanc avec lequel Philippe Couillard a cousu son allocution était particulièrement visible. Néanmoins, ça fonctionne. (Pepin, 2014)

À l'occasion de leur campagne électorale respective, Topp et Couillard ont chacun fait l'objet d'un article attirant l'attention sur leurs compétences linguistiques. Topp visait le poste de chef du Nouveau Parti démocratique (NPD) du Canada tandis que Couilllard aspirait au titre de Premier ministre du Québec. Ces exemples attestent du fait que les récepteurs d'un discours politique ne réagissent pas toujours aux anglicismes de la même façon. Parfois, les anglicismes répondent aux normes de la communauté linguistique, alors qu'à d'autres moments, ils s'en écartent. Les propos apparemment contradictoires des journalistes laissent croire soit que la norme n'est pas adoptée, consciemment ou non, par tous les membres de la communauté, soit que la norme varie en fonction du contexte d'élocution. Par exemple, dans les cas décrits, la présence d'anglicismes, c'est-à-dire d'atteintes à la norme, dans la bouche d'une personne ayant grandi dans un milieu bilingue ne remet pas en question la qualité du français du locuteur (M. Topp s'exprime parfaitement dans les deux langues [...]) ; par ailleurs, on reproche ses anglicismes à un francophone et on y voit une forme de relâchement linguistique (et de démagogie).

N'oublions pas que pour certains auteurs, dont Bernard Saint-Jacques (1990), les anglicismes sont associés au registre populaire. C'est pourquoi plusieurs ouvrages de référence répertorient et corrigent les formes interférentes (par exemple, Dagenais, 1984; Dubuc, 2000 ; Forest et al., 1998; Parmentier, 2006; De Villers, 2015; Cardinal et al., 2009). En vertu de sa mission de francisation, l’Office québécois de la langue française condamne lui aussi l'usage de certains anglicismes à l'écrit et recommande des solutions de rechange aux Québécois d'expression française. Toutefois, malgré leur mauvaise presse, les anglicismes font partie du quotidien des Québécois, comme le montre l'exemple du discours de Philippe Couillard.

Jusqu'à maintenant, la recherche s'est peu intéressée au contact des langues dans les discours en français des politiques du Canada et du Québec. La présente contribution vise à combler en partie cette lacune. Pour mener notre recherche à bien, nous traiterons des anglicismes dans les discours politiques. 


\section{Notre étude}

Selon Hélène Cajolet-Laganière, Marie-France Langlois et Pierre Martel (2001), collaborateurs à la première édition du dictionnaire québécois Usito, les emprunts critiqués à l'anglais font partie des spécificités linguistiques québécoises. Pour Martel et Cajolet-Laganière (1996), les anglicismes représentent également la manifestation d'une «norme spécifique au Québec » (dans la perspective de recherche d'une norme québécoise). Peut-être parce qu'elle symbolise la domination linguistique vécue par les nations canadienne-française et québécoise, cette retombée inévitable du phénomène de contact des langues a fait l'objet de nombreuses études, notamment en sociolinguistique (Bouchard, 2002 ; De Villers, 2005 ; Larrivée, 2009), en lexicologie (Poirier, 1995; Courbon et Paquet-Gauthier, 2014), en linguistique appliquée (Mareschal, 1989, 1992) et en traductologie (Darbelnet, 1968; Brunette, 1997).

Notre objectif est de jeter les bases d'une compréhension accrue de l'usage des anglicismes chez les hommes et les femmes politiques du Canada, du point de vue de la traductologie. Ce regard traductologique distingue cette exploration des travaux antérieurs. Les questions suivantes ont guidé notre recherche: existe-t-il une différence éloquente entre la proportion d'anglicismes (par rapport à l'ensemble du discours) dans des textes français du gouvernement fédéral du Canada, majoritairement issus de la traduction, et celle qu'on dégage des discours d'expression française prononcés sur la scène politique du Québec? À partir de cette analyse, quels sont les constats qui s'imposent à propos de la norme linguistique du Québec et du Canada?

Certains auteurs (Bouchard, 1998) ${ }^{1}$ vont jusqu'à affirmer que traquer l'anglicisme a pris les allures d'une véritable obsession nationale au Québec. De même lit-on en quatrième de couverture du Traducteur averti (Lavallée, 2005) :

Au-delà de la chasse aux anglicismes qui domine depuis longtemps au Québec le travail et la formation des traducteurs, François Lavallée considère son travail de traducteur et d'enseignant comme une «chasse aux tours idiomatiques», cette fois non pas comme on fait la chasse aux bisons, mais comme on fait la chasse aux papillons : pour enrichir sa collection.

C'est entre autres ce qui nous amène à poser l'hypothèse qu'il y aura moins d'anglicismes au fédéral qu'au provincial. En effet, le corpus fédéral est essentiellement composé de textes traduits vers le français. Or, sans doute parce qu'ils les connaissent, les traducteurs évitent davantage les anglicismes que ne le font les professionnels de la politique. On peut donc s'attendre à ce que le niveau de la langue du corpus de l'administration fédérale s'en trouve

1. L'édition de 1998 s'intitule La langue et le nombril. Histoire d'une obsession québécoise, celle de 2002, La langue et le nombril. Une histoire sociolinguistique du Québec. 
relevé. Cette affirmation repose sur notre connaissance intime des cursus de traduction des universités du Canada, de même que sur celle des pratiques traductives mises en œuvre et imposées au sein de l'administration centrale. En raison du bilinguisme officiel pratiqué au Canada et des conséquences linguistiques de cette politique, c'est-à-dire des nombreuses possibilités d'interférences, la formation des traducteurs (anglais-français ou français-anglais) inclut partout des cours de linguistique normative ou de français ou d'anglais correctif.

Ont donc été retenus les anglicismes, ce type de dérogation à la norme du français de référence ayant été attesté par Antidote. Par anglicisme, nous entendons tout report dans le français d'une forme linguistique de l'anglais. Notre analyse portera sur toutes les formes d'anglicismes repérables dans un texte, ce qui exclut les anglicismes phonétiques, mais inclut, notamment, les anglicismes lexicaux, sémantiques et syntaxiques.

Pour l'instant, notre examen se limite aux anglicismes. Il s'agit donc d'établir la fréquence relative de ces écarts à la norme dans les discours en français prononcés au fédéral et au provincial. Pour ce faire, nous avons calculé les formes répertoriées (en nombre) pour les comparer à la totalité du corpus, qu'il s'agisse de textes lus ou improvisés. À partir de ces résultats, nous tenterons de tirer des conclusions sur la présence des anglicismes dans les discours des politiques du Canada et du Québec.

\section{Méthodologie}

\section{Le corpus}

La présente étude se ramène à l'analyse d'un corpus couvrant la période 20102012 et constitué essentiellement d'extraits aléatoires de discours politiques. Ce corpus est composé des allocutions lues ou improvisées en réponse au Discours du Trône à la Chambre des communes (le fédéral) de même que les allocutions s'intégrant dans le débat qui suit l'Allocution d'ouverture à l'Assemblée nationale du Québec (le provincial). Dans ce dernier cas, la seule langue officielle est le français. Nous avons travaillé à partir de publications dites officielles, c'est-à-dire émanant des administrations concernées. Les retranscriptions proviennent donc toutes des publications officielles des gouvernements étudiés, à une exception près : lorsqu'il y avait de grandes divergences entre la retranscription officielle et l'enregistrement, nous avons également fourni notre propre retranscription.

L'analyse du corpus nous a amenées à mettre en parallèle les anglicismes repérés dans les discours traduits de l'anglais vers le français (gouvernement fédéral) et dans les discours originaux français (gouvernement provincial du 
Québec et gouvernement fédéral du Canada). Pour repérer les anglicismes, nous avons réalisé une analyse traductologique du corpus, conjuguée à une analyse semi-automatique des textes, effectuées à l'aide d'Antidote 8, logiciel intégrant à la fois un correcteur linguistique et plusieurs dictionnaires et guides de la langue française. Le recours à Antidote assure notamment le caractère reproductible de notre expérience. Lors de la révision humaine, les chercheuses ont utilisé un ensemble de ressources sur la langue, dont le manuel de traduction La traduction raisonnée (Delisle, 2013), les dictionnaires usuels de langue française ou anglaise et les dictionnaires québécois tels que le Multidictionnaire (De Villers, 2015), les dictionnaires d'Antidote (Druide informatique, 2012) et Usito (Cajolet-Laganière, Martel, 2014).

Le corpus compte 90149 mots au total, soit 45110 mots au fédéral et 45039 au provincial. Lors de la constitution du corpus, nous avons distingué les sources lues des sources improvisées parce que l'improvisation, en raison de sa nature spontanée, ne laisse pas place à la révision de la qualité de la langue avant la prestation. Nous constatons que le corpus fédéral est surtout lu (près de 26000 mots lus, ce qui représente près de $58 \%$ du corpus) alors que le corpus provincial, surtout improvisé (près de 29000 mots improvisés, ce qui représente près de $64 \%$ du corpus). Évidemment, les politiques qui ont improvisé leur discours n'ont pas été en mesure d'utiliser un correcteur informatique. Seuls les discours lus auraient pu être précorrigés, avec ou sans le recours à Antidote. C'est donc dire qu'avec les discours rédigés à l'avance, on peut avancer des hypothèses à propos de l'importance, pour les locuteurs, d'éviter les interférences linguistiques et les fautes de langue en général. Si certains textes lus par les politiques contiennent des erreurs repérées par le logiciel Antidote, on pourra conclure que le logiciel en question n'a pas été utilisé.

On remarquera aussi que d'un corpus à l'autre, le nombre de textes diffère. Ainsi, les parlementaires de l'Assemblée nationale disposent de plus de temps pour présenter leurs idées, parce qu'ils sont moins nombreux qu'à la Chambre des communes. Ces réalités expliquent les divergences quant au nombre de textes (et d'allocuteurs) dans les deux corpus : 54 pour le fédéral et 14 pour le provincial. Ici, c'est le nombre de mots qui a constitué le dénominateur commun entre les deux corpus.

Nous tenons à préciser les raisons motivant le choix d'un corpus datant de peu : d'une part, dans les territoires francophones du Canada et du Québec, comme partout ailleurs, il arrive souvent que l'anglicisme d'hier soit, demain, légitimement intégré au lexique de la langue touchée; d'autre part, nous avons utilisé un outil de détection, Antidote, qui fait souvent l'objet de mises à jour. À un outil à jour, il était important de faire correspondre un corpus récent. Soulignons encore que le recours à un outil de contrôle détectant les anglicismes présente l'avantage de garantir une certaine objectivité à l'opération de dépouillement en gardant les auteures de l'étude de toute perspective 
hypernormative. Tout correcteur informatique ayant ses limites, nous avons également procédé à un dépouillement «humain » afin de repérer les anglicismes et les québécismes ayant échappé au balayage du logiciel.

\section{Contextualisation}

Même si les parlements du Québec et du Canada sont l'un et l'autre héritiers de la tradition britannique, leur façon de traiter le discours du représentant de la Couronne n'est pas la même. Ainsi, à l'Assemblée nationale du Québec, la terminologie employée à propos du discours élimine le plus possible les allusions à la monarchie. L'ancien Discours du Trône, prononcé par le lieutenantgouverneur général, est aujourd'hui «l'allocution d'ouverture». De plus, cette allocution est immédiatement suivie du «discours d'ouverture » prononcé par le premier ministre du Québec. C'est lors du discours d'ouverture que le premier ministre expose le programme de son gouvernement. Manifestement plus important que l'allocution d'ouverture, le discours du premier ministre attire toute l'attention des médias. Le débat qui s'ensuit dure vingt-cinq heures. Le chef de l'opposition officielle est le premier parlementaire à prendre la parole lors du débat. Ensuite, les députés volontaires s'expriment à tour de rôle. Ils sont limités à un discours, mais au cours duquel ils sont libres d'aborder quelque sujet que ce soit (Direction des travaux parlementaires, 2014). Les députés s'expriment dans la langue de leur choix. Concrètement, à Québec, le français, officiel, est majoritairement utilisé, l'anglais, un peu, et les autres langues, très peu.

À la Chambre des communes, c'est-à-dire au gouvernement fédéral, le Discours du Trône a gardé son nom et il constitue un moment fort de la vie parlementaire. C'est le gouverneur général (ou le souverain, s’il est sur place), qui lit le discours, mais ce sont les conseillers du Premier ministre qui en rédigent l'essentiel. Ce discours décrit les vues du gouvernement sur l'état du pays. Le gouverneur général y donne aussi une indication du programme du gouvernement. Depuis le $x x^{e}$ siècle, le discours est prononcé en français et en anglais. Sa traduction est donc effectuée avant la lecture officielle et le débat de portée générale qui suit est lancé par deux discours clefs : la motion sur l'Adresse en réponse au discours du trône et l'appui de la motion sur l'Adresse. Ces discours sont généralement prononcés par des députés de l'arrière-ban du parti au pouvoir, récemment élus (Services de la procédure. Chambre des communes, 2009).

À la Chambre des communes, les députés s'expriment dans la langue officielle de leur choix. Depuis 1959, le Parlement du Canada dispose d'un service d'interprétation simultanée en français et en anglais. Dans la vaste majorité des cas, à l'Assemblée nationale du Québec, il n'y a pas d'interprétation simultanée. 
En politique, il est parfois difficile d'établir avec certitude qui est l'auteur d'un discours et notre corpus ne fait pas exception. Dans ses travaux, Chantal Gagnon $(2006,2010)$ a analysé les archives d'anciens premiers ministres au fédéral et elle a démontré qu'au Canada, les discours politiques du Premier ministre du gouvernement fédéral sont souvent écrits par une équipe de conseillers bilingues. Il arrive même que certains discours soient rédigés partiellement en français et partiellement en anglais. En règle générale, il est impossible de déterminer avec certitude la langue d'origine d'un discours, puisque les discours sont présentés comme deux originaux par le gouvernement fédéral (Gagnon, 2006). Cependant, puisque l'anglais est la langue dominante au gouvernement fédéral, on peut avancer l'hypothèse que les discours sont très souvent rédigés en anglais, particulièrement lorsque le politique est anglophone.

Les discours du corpus sont traduits par des fonctionnaires après le débat. Plus précisément, au gouvernement fédéral, les débats sont retranscrits et traduits de nuit, afin que les députés y aient accès le lendemain matin. La traduction est assurée par des traducteurs professionnels du service de traduction du gouvernement, le Bureau de la traduction. L'ouvrage réunissant les discours de la Chambre des communes s'intitule en français Débats de la Chambre des communes, ou "Hansard». En anglais, la publication des débats a pour titre House of Commons Debates, ou «Hansard». Selon la documentation de la Chambre des communes, le Hansard constitue une «transcription corrigée des délibérations de la Chambre et des Comités pléniers» (Services de la procédure. Chambre des communes, 2010). Soulignons aussi que selon la Direction de la traduction parlementaire, que nous avons consultée, les traducteurs ont accès à Antidote dans le cadre de leur travail. Cependant, il semble que ce correcteur informatique s'arrime mal avec les autres outils de traduction des fonctionnaires. Ainsi, l'utilisation du correcteur exige une étape supplémentaire dans le processus de traduction, étape laissée à la discrétion des traducteurs.

À l'Assemblée nationale, les discours ne sont pas traduits, à l'exception notable du discours sur le budget, traduit vers l'anglais. Cette traduction ne figure cependant pas dans la publication officielle des débats, appelée Journal des débats. La transcription fidèle est au cœur de la politique éditoriale du Journal. Ainsi, «aucune correction visant à modifier le style ou le vocabulaire n'est autorisée» (Assemblée nationale du Québec, 2014). Si des députés de l'Assemblée s'expriment dans une langue autre que le français ou l'anglais, leurs propos ne seront pas retranscrits. 


\section{Analyse des résultats}

\section{Analyse quantitative}

De façon absolue, les discours prononcés dans l'enceinte de la Chambre des communes à Ottawa comportent davantage d'anglicismes (71) que ceux des députés dits provinciaux (51). On le remarque notamment en consultant le tableau 1, qui présente une compilation de tous les anglicismes répertoriés dans le corpus. N'oublions pas que la majorité des textes français du corpus fédéral ont fait l'objet d'une traduction. On l'a vu, les traducteurs professionnels sont formés pour éviter les interférences linguistiques. Il est donc étonnant qu'ils commettent plus d'anglicismes que les politiques francophones du Québec, qui n'ont, pour la plupart, pas reçu une telle formation.

Tableau 1. Compilation de tous les anglicismes

\begin{tabular}{l|c|c}
\hline & Nombre de mots du corpus & Anglicismes à l'écrit (tous) \\
\hline Provincial & 45039 & 51 \\
\hline Fédéral & 45110 & 71 \\
\hline
\end{tabular}

Tableau 2. Compilation des anglicismes, analyse semi-automatique

\begin{tabular}{l|c|c}
\hline & Nombre de mots du corpus & $\begin{array}{c}\text { Anglicismes à l'écrit } \\
\text { repérés par Antidote }\end{array}$ \\
\hline Provincial & 45039 & 33 \\
\hline Fédéral & 45110 & 28 \\
\hline
\end{tabular}

Tableau 3. Compilation des anglicismes, dépouillement « humain »

\begin{tabular}{l|c|c}
\hline & Nombre de mots du corpus & $\begin{array}{c}\text { Anglicismes à l'écrit non } \\
\text { repérés par Antidote }\end{array}$ \\
\hline Provincial & 45039 & 18 \\
\hline Fédéral & 45110 & 43 \\
\hline
\end{tabular}

La différence visible quant au nombre d'anglicismes dans les corpus du fédéral et du provincial s'estompe dans le tableau 2. Dans ce dernier tableau, seuls sont répertoriés les anglicismes relevés par le logiciel Antidote. La différence entre le tableau 1 et le tableau 2 mérite qu'on s'y attarde. En effet, les anglicismes retenus par Antidote sont plus répandus ou moins complexes que ceux repérés sans l'aide du logiciel. Parmi les anglicismes plus complexes figurent 
les anglicismes syntaxiques, les anglicismes de fréquence et les cas d'adjectivite ${ }^{2}$, qui nécessitent une analyse contextuelle plus approfondie que des cas d'anglicismes sémantiques courants. C'est donc sous l'effet d'une analyse fouillée que les anglicismes du fédéral se sont révélés. Voici deux exemples représentatifs, lus par des députés du gouvernement fédéral :

(1) Because of prorogation, because of this government's contempt for Canada's institutions, we have a democratic deficit to go with our budget deficit. À cause de la prorogation, à cause du mépris de ce gouvernement pour les institutions du Canada, nous avons un déficit démocratique en plus d'un déficit budgétaire. (Michael Ignatieff, Chambre des communes, traduction de l'anglais)

(2) On the contrary, this government is ignoring the very clear expression of the will of this House regarding handing over the documents that our committee on the Afghanistan mission needs to do its very important work. Au contraire, ce gouvernement ignore la volonté très claire de cette Chambre quand il s'agit de la production de documents nécessaires au travail si important de notre comité sur la mission en Afghanistan. (Dominic Leblanc, Chambre des communes, traduction de l'anglais)

Dans ces extraits, on remarque que la traduction du déictique this a entraîné des calques difficilement repérables par le logiciel. Selon J. Delisle (2013, p. 398), le français présente la spatialisation de façon abstraite dans ce type de situation, contrairement à l'anglais : «Les comparatistes ont remarqué que, dans le domaine de la spatialisation (situation du locuteur dans l'espace), il existe un écart net entre le dénuement (économie) du français et la richesse des moyens dont dispose l'anglais pour marquer ce genre de rapports. »

Précisons encore une fois que ce qui différencie le corpus du fédéral du corpus du provincial, c'est la traduction. Le corpus fédéral se compose essentiellement de textes traduits alors que le provincial, de textes originaux. Sans doute le plus grand nombre d'anglicismes au fédéral s'explique-t-il par le processus du transfert linguistique, de l'anglais vers le français. Comme l'a écrit le Français Maurice Pergnier (1993, p. 226) : «Il est bien connu, par exemple, qu'un sujet bilingue est plus vulnérable aux interférences lorsqu'il est en situation de traduction, qu'il ne l'est lorsqu'il utilise ses deux langues indépendamment l'une de l'autre. » On pourrait aussi affirmer que le processus cognitif de la traduction donne lieu à des types d'anglicisme particuliers. Ainsi, jusqu'à un certain point, l'anglicisme compte parmi les caractéristiques textuelles des discours traduits à la Chambre des communes. Mais jusqu'à un certain point seulement. Car, toutes proportions gardées, il semble bien que la différence n'est pas révélatrice, les anglicismes comptant pour 0,113 \% dans le corpus compilé à Québec contre 0,157 \% dans celui monté avec les discours d’Ottawa.

2. «Emploi fautif de l'adjectif qualificatif comme adjectif de relation ou épithète de relation, habituellement sous l'influence de l'anglais» (TERMIUM Plus, la banque de données terminologiques et linguistiques du gouvernement du Canada, http://www.btb.termiumplus.gc.ca). 


\section{Analyse qualitative}

Notre étude du corpus montre que les emprunts directs de l'anglais sont mieux acceptés au gouvernement provincial qu'au gouvernement fédéral, même si dans les deux cas, ils sont peu fréquents. Les emprunts directs, dits aussi emprunts lexicaux, correspondent au report intégral (forme et sens) ou partiel (forme ou sens seulement) d'une unité lexicale étrangère en français (Loubier, 2011). Considérons les trois exemples ci-dessous, improvisés par les députés du Québec:

(1) [La circonscription de] Chauveau, M. le Président, c'est l'industrie récréotouristique, c'est le jardin, c'est le «playground », comme on diten anglais, c'est le terrain de jeu des gens de Québec pour la bretelle nord, mais c'est aussi, M. le Président, un secteur manufacturier qui est toujours présent. (Gérard Deltell, Assemblée nationale, original)

(2) Dans mon propre comté, M. le Président, je pourrais vous nommer une dizaine d'entreprises qui aujourd'hui embauchent des gens, bien, qui travaillent dans le Nord, dans un contexte de "fly-in / fly-out» - on y va, on revient - des entreprises qui fabriquent notamment des pièces de bâtiment dans mon comté, au Témiscouata, chez Produits PBM, qui ont de l'emploi grâce au Nord. (Jean D'Amour, Assemblée nationale, original)

(3) 800 emplois, 800 jobs directement dans une communauté, comme ancien maire de Rivière-du-Loup, comme député d'une région, je peux comprendre à quel point 800 emplois, c'est dur à supporter, que c'est un dur coup pour l'économie, c'est un coup de couteau en plein cœur, M. le Président. (Jean D'amour, Assemblée nationale, original)

Dans deux des trois exemples, les politiques du gouvernement provincial ont ressenti le besoin de préciser leur pensée en français. C'est donc que s'il est envisageable d'utiliser des mots anglais peu connus dans un discours de l'Assemblée nationale, il faut y ajouter un vernis français. Le troisième exemple présente un anglicisme connu et souvent toléré hors Québec, job. Ici, le député renforce un mot français à l'aide d'un emprunt qui lui sert d'augmentatif, comme si, pour clore son argumentation, le politique avait besoin de changer de registre et de langue. Selon les dictionnaires Antidote et Usito, job, généralement au féminin au Québec, appartient au registre familier. Pour le Multidictionnaire, job n'est jamais admis au Québec.

Si on exclut l'anglicisme leadership critiqué par Antidote et Usito, mais accepté ailleurs dans le monde francophone et dans le Multidictionnaire, on ne relève aucun anglicisme d'emprunt dans le corpus du Compte rendu des débats de la Chambre des communes. Au fédéral, les emprunts n'ont donc pas droit de cité à l'écrit. Cependant, en comparant la version officielle à l'en- 
registrement audio du débat de la Chambre des communes, on constate des différences entre les deux productions. L'exemple suivant, improvisé par un député fédéral, l’illustre bien :

(4) Version orale (transcription) : Une chose est certaine, Monsieur le Président, c'est qu'on fait le travail, on fait la job.

Version officielle du Compte rendu, révisée : Chose certaine, on fait le travail. (Bernard Généreux, Chambre des communes, original)

Le réviseur du gouvernement fédéral a fait disparaître l'emprunt direct lors de la transcription. Au provincial, le même type d'exemple a été laissé tel quel. Le réviseur du fédéral a aussi retravaillé la formulation afin qu'elle soit plus ramassée. Les normes observées au gouvernement fédéral rejoignent les recherches de Geneviève Mareschal $(1989,1992)$ et de Marie-Éva de Villers (2005), qui ont toutes deux révélé qu'au Québec, dans la presse, les emprunts directs sont peu nombreux. Les travaux de Chantal Bouchard, qui s'est penchée sur l'histoire des normes sociolinguistiques au Québec, pourraient nous éclairer sur ce phénomène. C. Bouchard (1989, p. 87) écrit : "La contamination du français par l'anglais apparaît aux francophones du Québec, du moins à ceux qui s'expriment sur la question, comme un symptôme de leur impuissance, aussi le rejet en est-il violent ».

Notre étude montre que les réviseurs du fédéral ne corrigent pas seulement les emprunts directs du corpus. En effet, les anglicismes syntaxiques et sémantiques font aussi l'objet d'interventions. Voici deux exemples représentatifs :

(5) Version orale (transcription) : Le seul parti qui a résisté à la reconnaissance de la nation québécoise était le Bloc. Et pourquoi, Madame la Présidente, est-ce que le Bloc a résisté cette idée?

Version officielle du Compte rendu, révisée : Le seul parti qui a résisté à la reconnaissance de la nation québécoise était le Bloc. Pourquoi le Bloc a-t-il résistéà cette idée? (Stephen Harper, Chambre des communes, original)

(6) Version orale (transcription) : On a un gouvernement qui a peur de l'imputabilité, qui n'est pas intéressé à renforcer la démocratie canadienne. Et au contraire, qui ignore la volonté très claire de cette chambre quand il s'agit de la production de documents nécessaires pour le travail si important que notre comité sur la question en Afghanistan.

Version officielle du Compte rendu, révisée : On a devant nous un gouvernement qui a peur de l'obligation de rendre des comptes et qui n'est pas intéressé à renforcer la démocratie canadienne. Au contraire, ce gouvernement ignore la volonté très claire de cette Chambre quand il s'agit de la production de documents nécessaires au travail si important de notre comité sur la mission en Afghanistan. (Dominic LeBlanc, Chambre des communes, original) 
Le député acadien Dominic LeBlanc (exemple 6) a glissé un anglicisme sémantique dans son discours improvisé en français. À l'écrit, il n'y a plus de trace du calque. Quant au discours improvisé en français de l'ancien Premier ministre du Canada, Stephen Harper (exemple 5), il contient un anglicisme syntaxique qui disparaît dans la version officielle.

Les exemples présentés révèlent qu'au gouvernement fédéral, il y a deux normes langagières, celle des fonctionnaires, qui dressent le compte rendu en français, et celle des politiques. La norme des fonctionnaires est assimilable à une règle. Les ouvrages publiés par le gouvernement fédéral témoignent de cette norme. Le terme «imputabilité», par exemple, figure parmi les expressions à éviter dans Clefs du français pratique et dans Termium, deux publications en ligne du Bureau de la traduction. Quant à la norme des politiques, elle est assimilable à un modèle. Et on revient à C. Le Bart : «Les politiques [...] sont contraints à parler comme tout le monde s'attend à les entendre parler. » Pour les politiques, il ne s'agit donc pas de respecter les recommandations documentées du gouvernement fédéral en matière de langue française, mais bien de respecter la norme non dite et non écrite du français parlé par la communauté des électeurs.

Le cas «imputabilité» représente bien la relation asymptotique qui existe entre les deux normes langagières au fédéral. En effet, en 2006, les fonctionnaires avaient réussi à convaincre le Cabinet du Premier ministre, par l'intermédiaire des médias et d'un parti de l'opposition, de modifier le projet de loi sur l'imputabilité afin de le renommer "Loi sur la responsabilité». Malgré les débats en chambre et la polémique dans les journaux, quatre années plus tard, des parlementaires utilisent encore «imputabilité » de façon fautive (selon les normes écrites du gouvernement fédéral).

Les exemples (1) à (6) de cette section présentent tous des extraits improvisés. Or les extraits lus contiennent eux aussi de nombreux anglicismes. Voici deux exemples de textes lus :

(7) Déjà, l'an dernier, ce gouvernement a coupé 1 milliard de dollars au Québec en péréquation, et ce, malgré les promesses de ce gouvernement de ne pas en modifier la formule. (Gilles Duceppe, Chambre des communes, original)

(8) Pour Montréal, le gouvernement mettra l'emphase sur quatre priorités [...]. (Pauline Marois, Assemblée nationale, original)

Ces deux extraits ont été lus par des chefs québécois de partis indépendantistes, soit le Bloc québécois au fédéral et le Parti québécois au provincial. Pour ces deux partis, et pour les chefs qui les dirigeaient, la question de la langue française est très importante, et la qualité de la langue l'est tout autant. Que ces chefs n'aient pas évité ces anglicismes sémantiques - pourtant facilement repérable avec un bon dictionnaire québécois - montre que du point de vue des politiques, les anglicismes sémantiques répondent aux normes de la communauté linguistique. 
Les pratiques de l'Assemblée nationale et de la Chambre des communes diffèrent à bien des égards, on l'a vu. À l'écrit, la transcription des débats des deux parlements diverge énormément, puisque du côté québécois, il s'agit d'une transcription textuelle tandis que du côté canadien, les discours sont révisés. Pourquoi la révision au fédéral ? Sans doute pour les mêmes raisons que l'on traduit vers le français : pour assurer une communication efficace et pour protéger une certaine conception de la langue française. Cependant, cette révision donne l'impression que les députés de la Chambre des communes s'expriment dans un français relativement dénué d'interférences linguistiques. On remarque le rapport de force entre le français attendu et le français réel. À certains égards, il y a donc aplanissement des différences culturelles et institutionnelles du français de la Chambre des communes (voir aussi Brunette, 2002). Si ce rapport de force semble exclusif au gouvernement fédéral, c'est que la communauté francophone y est nettement minoritaire et le risque d'anglicisation, nettement plus fort. Plus la situation d'interférence linguistique est appréciable, plus la norme semble restrictive.

Nous avons établi que les francophones du Canada, et particulièrement les Québécois, accordent une grande importance à la question des anglicismes. C'est la raison pour laquelle un logiciel de correction comme Antidote rectifie non seulement la grammaire et la syntaxe, mais aussi les fautes attribuables à des situations d'interférence linguistique. Or, notre étude montre que même lorsque les discours sont rédigés à l'avance en français, les politiques n'utilisent pas d'outils de correction pour éviter les anglicismes. Par exemple, dans le corpus du provincial, tous les discours lus à l'Assemblée nationale par des politiques contiennent au moins un anglicisme détecté par Antidote. Au gouvernement fédéral, des discours lus en français à la Chambre des communes présentent également des anglicismes repérés par Antidote, notamment dans les discours du premier ministre du Canada et dans le discours du chef du Parti libéral du Canada. La non-utilisation du logiciel est assez répandue dans les deux parlements pour qu'on affirme que les politiques ne sentent pas la nécessité de mettre en place une stratégie d'évitement des anglicismes. Les anglicismes sont acceptés dans les parlements du Québec et du Canada et ils font partie de la norme. Cependant, cette tolérance ne s'étend pas nécessairement à tous les types d'anglicisme. Nous avons vu qu'à l'écrit, au fédéral, des réviseurs gomment la présence d'emprunts directs utilisés par des députés d'expression française. Par ailleurs, au provincial, les emprunts directs n'ont de place qu'à l'oral: dans les discours lus à l'Assemblée nationale, on ne retrouve pas d'emprunt direct. La norme porte donc sur les anglicismes visibles. Les anglicismes sémantiques ou syntaxiques, généralement plus difficiles à repérer pour les non-spécialistes, ne sont pas évités aussi systématiquement que les emprunts directs. De ce point de vue, nos résultats vont dans le sens des travaux de M.-É. de Villers (2005) et de G. Mareschal $(1989,1992)$, qui concluent 
que dans les quotidiens nationaux de langue française, les Québécois s’abstiennent de recourir aux emprunts directs à l'anglais.

Notre analyse qualitative a notamment révélé que les anglicismes sont très peu fréquents dans les corpus (0,113\% dans le corpus provincial contre 0,157\% dans le corpus fédéral). Certains facteurs abordés dans cet article contribuent à l'explication de ce résultat. Ainsi, au gouvernement fédéral, les discours sont tous relus par des professionnels de la langue. De plus, nous avons évoqué que les anglicismes sont associés, au Québec, à la question du registre de langue. Or, les députés d'un parlement appartiennent à une classe sociale favorisée, qui s'exprime dans une langue plus soutenue que la moyenne.

Nous avions posé l'hypothèse que les traducteurs professionnels seraient davantage en mesure d'éviter les anglicismes que les politiques du Québec. II semble que ce ne soit pas le cas. En fait, les résultats de notre recherche suggèrent que la situation d'interférence linguistique vécue par les traducteurs est telle qu'ils ne sont pas à même d'enrayer les anglicismes de leurs écrits. Les traducteurs de notre corpus, pourtant formés et expérimentés, n'arrivent pas à respecter les normes édictées par le Bureau de la traduction sur la façon de traduire certaines difficultés de l'anglais. À leur décharge, les conditions de travail de ces traducteurs sont difficiles : les traductions doivent être effectuées rapidement et de nuit. Pour confirmer nos résultats, il faudrait à la fois élargir le corpus parlementaire et le comparer à des traductions effectuées par le Bureau de la traduction dans des délais raisonnables. Il ne reste plus qu'à souhaiter que d'autres chercheurs s'intéresseront à la question des anglicismes dans les discours politiques3.

\section{Références}

Assemblée nationale du QuÉBec, 2014, L'ABC de l'Assemblée. L'organisation des travaux de l'Assemblée, Québec, http://www.assnat.qc.ca/fr/abc-assemblee/organisation-travaux-assemblee/ publications.html\#JDD (consulté le 15 mars 2016).

Bouchard Chantal, 1989, "Une obsession nationale : l'anglicisme», Recherches sociographiques, vol. 30, $\mathrm{n}^{\circ} 1, \mathrm{p} .67-90$.

- 1998, La langue et le nombril. Histoire d'une obsession québécoise, Saint-Laurent, Québec, Fides.

- 2002, La langue et le nombril. Une histoire sociolinguistique du Québec, 2 édition, Saint-Laurent, Québec, Fides.

BRUNETtE Louise, 1997, Contribution à la pédagogie de la révision en pays bilingue. Le cas du Canada, Villeneuve d'Ascq, Presses universitaires du Septentrion.

3. Les auteures tiennent à remercier le doctorant Esmaeil Kalantari (Université de Montréal) pour la qualité de son travail. Par ailleurs, cet article a profité du soutien financier du Fonds de recherche du Québec, société et culture. 
- 2002, "Normes et censure : ne pas confondre», $\Pi R$ : traduction, terminologie, rédaction, vol. $15, \mathrm{n}^{\circ} 2$, p. 223-233.

CAjolet-Laganière Hélène, LANGlois Marie-France, MARTel Pierre, 2001, "La pertinence culturelle et linguistique dans les outils linguistiques informatisés couramment utilisés au Québec», Revue belge de philologie et d'histoire, vol.79, n³, p. 861-881.

CAJOlet-Laganière Hélène, Martel Pierre, 2014, Usito, en ligne : http://www.usito. com/dictio/ (consulté le 15 mars 2016)

Cardinal Philippe, Melançon Christiane, Hébert Christine, Laviolette-Chartrand Marie-Paule, 2009, Le vocabulAIDE. Influences de l'anglais, vraies et prétendues, et usages en transition, Ottawa, Presses de l'Université d'Ottawa.

Courbon Bruno, PAquet-Gauthier Myriam, 2014, «Faux amis / vrais ennemis. Réutilisations de la notion d'anglicisme dans le discours métalinguistique au Québec», Le discours et la langue, vol. 6, $\mathrm{n}^{0} 1$, p.143-173.

DAGENAIS Gérard, 1984, Dictionnaire des difficultés de la langue française au Canada, Boucherville, Éditions françaises.

DARBELNET Jean, 1968, «La traduction, voie ouverte à l'anglicisation », Culture vivante, $n^{\circ} 7-8$, p. 39-45.

DelisLe Jean, 2013, La traduction raisonnée. Manuel d'initiation à la traduction professionnelle, anglais, français : méthode par objectifs d'apprentissage, Ottawa, Presses de l'Université d'Ottawa.

DIRECTION DES TRAVAUX PARLEMENTAIRES, 2014, «Règlement et autres règles de procédures», Direction des communications, des programmes éducatifs et de l'accueil, Québec, Assemblée nationale, http://www.assnat.qc.ca/fr/publications/fichereglement-assemblee.html (consulté le 15 mars 2016)

Druide informatique, 2012, Antidote 8, Correcteur, Dictionnaires, Guides, Montréal, Druide informatique.

DuBuc Robert, 2000, En français dans le texte, Brossard, Linguatech.

Forest Constance, Colpron Gilles, Boudreau Denise, 1998, Le Colpron. Le dictionnaire des anglicismes, Laval, Beauchemin.

GAGNON Chantal, 2006, "Language plurality as power struggle, or: Translating politics in Canada», Target. International Journal of Translation Studies, vol.18, nº 1 , p. 69-90.

- 2010, «When text and translation production meet. Translation in the Prime Minister’s Office », C. Schäffner, S. Bassnett éd., Political Discourse, Media and Translation, Cambridge, Cambridge Scholars Publishing, p. 164-177.

GIROUX Raymond, 2011, «Un Canadien errant», Le Soleil, vol. 115, n² 271, p. 12.

LARRIVÉE Pierre, 2009, Les Français, les Québécois et la langue de l'autre, Paris, L'Harmattan.

LAVALlÉE François, 2005, Le traducteur averti. Pour des traductions idiomatiques, Brossard, Linguatech.

Le BART Christian, 1998, Le discours politique, Paris, Presses universitaires de France. Loubier Christiane, 2011, De l'usage de l'emprunt linguistique, Montréal, Office québécois de la langue française. 
MARESCHAL Geneviève, 1989, Étude typologique et comparative de l'anglicisation et des anglicismes dans quatre aires de la francophonie, thèse de doctorat, Département de langues, linguistique et traduction, Université Laval.

- 1992, "L'influence comparée de l'anglais sur le français dans différentes aires géographiques francophones", Revue canadienne de linguistique appliquée, vol.14, no 1, p. 107-120.

MARTEL Pierre, CAJOLET-LAGANIÈRE Hélène, 1996, «Le français québécois. Une langue à plusieurs visages », Sommet. Le magazine de l'Université de Sherbrooke, vol. 9, n², http://www.usherbrooke.ca/catifq/fileadmin/sites/catifq/contributions/CAJOLET_Sommets.pdf (consulté le 15 mars 2016).

MARTel Pierre, Cajolet-Laganière Hélène, LANGlois Marie-France, 2001, "Les textes journalistiques québécois sont-ils "envahis" par les emprunts critiqués à l'anglais? ", Terminogramme, n $97-98$, p. 47-71.

PARMENTIER Michel, 2006, Dictionnaire des expressions et tournures calquées sur l'anglais, Sainte-Foy, Presses de l'Université Laval.

PEPIN Michel, 2014, "Couillard 2.0", Sur les collines. Le blogue politique de RadioCanada, http://blogues.radio-canada.ca/politique/2014/03/06/couillard-2-o/ (consulté le 15 mars 2016).

Pergnier Maurice, 1993, Les fondements sociolinguistiques de la traduction, Lille, Presses universitaires de Lille.

PoIRIER Claude, 1995, «Les variantes topolectales du lexique français. Propositions de classement à partir d'exemples québécois », M. Francard, D. Latin éd., Le régionalisme lexical, Louvain-la-Neuve, Duculot, p.13-56.

SAINT-JACQUES Bernard, 1990, "Le français québécois : langue de communication et symbole d'identité », N. Corbett éd., Langue et identité. Le français et les francophones d'Amérique du Nord, Québec, Presses de l'Université Laval, p. 229-244.

Services de LA PRoCÉdure. Chambre des COMmUnes, 2009, La procédure et les usages de la Chambre des communes, Ottawa, http://www.parl.gc.ca/procedure-booklivre/Document.aspx?sbdid=1CDFoCo9-DE93-4789-AA8D-67AFC1F658E2\&sbpidx $=1 \&$ Language $=F \& M o d e=1$ (consulté le 15 mars 2016).

- 2010, Publications parlementaires, Ottawa, http://www.parl.gc.ca/About/House/ compendium/web-content/c_g_parliamentarypublications-f.htm (consulté le 15 mars 2016).

VILlers Marie-Éva (de), 2005, Le vif désir de durer. Illustration de la norme réelle du français québécois, Montréal, Québec Amérique.

- 2015, Multidictionnaire de la langue française, Montréal, Québec Amérique.

\section{Corpus}

\section{Canada. Parlement. Chambre des communes}

3 mars 2010, Débat de la Chambre des communes, vol. 145, nº 138, $3^{\mathrm{e}}$ session, $40^{\mathrm{e}}$ législature. http://www.parl.gc.ca/HousePublications/Publication.aspx?Language= F\&Mode $=1 \&$ Parl $=40 \&$ Ses $=3 \&$ Docld $=5006735$ (consulté, comme les suivants, le 15 mars 2016). 
3 mars 2010, House of Commons Debates, ibid.

http://www.parl.gc.ca/HousePublications/Publication.aspx? Mode $=1 \&$ Parl=40\& Ses $=3 \&$ Docld $=5006735$ \& Language $=E$.

1. mars 2010, Débat de la Chambre des communes, ibid.

http://www.parl.gc.ca/HousePublications/Publication.aspx?Language=F\&Mode $=1 \&$ Parl $=40 \&$ Ses $=3 \&$ Docld $=5037709$.

1. mars 2010, House of Commons Debates, ibid.

http://www.parl.gc.ca/HousePublications/Publication.aspx?Mode=1\&Parl=40\& Ses $=3 \&$ Docld $=5037709$ \& Language $=E$.

3 juin 2011, Débat de la Chambre des communes, vol. 145, n² 2, $1^{\text {re }}$ session, $41^{\text {e législature }}$ http://www.parl.gc.ca/HousePublications/Publication.aspx?Doc $=2 \&$ Mode $=1 \& \mathrm{~Pa}$ $\mathrm{rl}=41 \& \mathrm{Pub}=$ Hansard \&Ses $=1$ \&Language $=\mathrm{F}$.

3 juin 2011, House of Commons Debates, ibid.

http://www.parl.gc.ca/HousePublications/Publication.aspx $?$ Doc $=2 \&$ Mode $=1 \& \mathrm{~Pa}$ $\mathrm{rl}=41 \& \mathrm{Pub}=$ Hansard $\&$ Ses $=1 \&$ Language $=\mathrm{E}$.

\section{Québec. Parlement. Assemblée nationale}

3. octobre 2012, Journal des débats de l'Assemblée nationale, vol. 43, nº 3, 1 $1^{\text {re }}$ session, $40^{\mathrm{e}}$ législature

http://www.assnat.qc.ca/fr/travaux-parlementaires/assemblee-nationale/40-1/ journal-debats/20121031/70025.html.

$1^{\text {er }}$ novembre 2012, Journal des débats de l'Assemblée nationale, ibid.

http://www.assnat.qc.ca/fr/travaux-parlementaires/assemblee-nationale/40-1/ journal-debats/20121101/70067.html.

7 novembre 2012, Journal des débats de l'Assemblée nationale, ibid.

http://www.assnat.qc.ca/fr/travaux-parlementaires/assemblee-nationale/40-1/ journal-debats/20121107/70447.html. 
